\title{
Causes of Medication Administration Errors in Hospitals: a Systematic Review of Quantitative and Qualitative Evidence
}

\author{
Richard N. Keers $\cdot$ Steven D. Williams $\cdot$ \\ Jonathan Cooke $\cdot$ Darren M. Ashcroft
}

Published online: 24 August 2013

(c) The Author(s) 2013. This article is published with open access at Springerlink.com

\begin{abstract}
Background Underlying systems factors have been seen to be crucial contributors to the occurrence of medication errors. By understanding the causes of these errors, the most appropriate interventions can be designed and implemented to minimise their occurrence.

Objective This study aimed to systematically review and appraise empirical evidence relating to the causes of medication administration errors (MAEs) in hospital settings.

Data Sources Nine electronic databases (MEDLINE, EMBASE, International Pharmaceutical Abstracts, ASSIA, PsycINFO, British Nursing Index, CINAHL, Health Management Information Consortium and Social Science Citations Index) were searched between 1985 and May 2013.
\end{abstract}

R. N. Keers $(\varangle)$. D. M. Ashcroft

Manchester Pharmacy School, NIHR Greater Manchester Primary Care Patient Safety Translational Research Centre, Manchester Academic Health Sciences Centre (MAHSC), University of Manchester, Manchester M13 9PT, UK e-mail: richard.keers@manchester.ac.uk

S. D. Williams

University Hospital of South Manchester NHS Foundation Trust, Manchester M23 9LT, UK

S. D. Williams · J. Cooke

Manchester Pharmacy School, University of Manchester, Manchester M13 9PT, UK

J. Cooke

Infectious Diseases and Immunity Section, Division of Infectious Diseases, Department of Medicine, Imperial College London, London SW7 2AZ, UK
Study Selection Inclusion and exclusion criteria were applied to identify eligible publications through title analysis followed by abstract and then full text examination. English language publications reporting empirical data on causes of MAEs were included. Reference lists of included articles and relevant review papers were hand searched for additional studies. Studies were excluded if they did not report data on specific MAEs, used accounts from individuals not directly involved in the MAE concerned or were presented as conference abstracts with insufficient detail.

Data Appraisal and Synthesis Methods A total of 54 unique studies were included. Causes of MAEs were categorised according to Reason's model of accident causation. Studies were assessed to determine relevance to the research question and how likely the results were to reflect the potential underlying causes of MAEs based on the method(s) used.

Results Slips and lapses were the most commonly reported unsafe acts, followed by knowledge-based mistakes and deliberate violations. Error-provoking conditions influencing administration errors included inadequate written communication (prescriptions, documentation, transcription), problems with medicines supply and storage (pharmacy dispensing errors and ward stock management), high perceived workload, problems with ward-based equipment (access, functionality), patient factors (availability, acuity), staff health status (fatigue, stress) and interruptions/distractions during drug administration. Few studies sought to determine the causes of intravenous MAEs. A number of latent pathway conditions were less well explored, including local working culture and highlevel managerial decisions. Causes were often described superficially; this may be related to the use of quantitative surveys and observation methods in many studies, limited 
use of established error causation frameworks to analyse data and a predominant focus on issues other than the causes of MAEs among studies.

Limitations As only English language publications were included, some relevant studies may have been missed.

Conclusions Limited evidence from studies included in this systematic review suggests that MAEs are influenced by multiple systems factors, but if and how these arise and interconnect to lead to errors remains to be fully determined. Further research with a theoretical focus is needed to investigate the MAE causation pathway, with an emphasis on ensuring interventions designed to minimise MAEs target recognised underlying causes of errors to maximise their impact.

\section{Background}

The reality that medical treatment can harm patients is one that the healthcare community has had to come to terms with over recent years [1]. In particular, adverse events associated with medication appear among the chief causes of this harm while patients reside in hospitals [2] and are known to be responsible for a large proportion of hospital admissions [3]. Preventable adverse drug events (ADEs) occurring during the medication use process in hospitals are associated with additional length of stay and healthcare costs [4]. Prescribing and drug administration appear to be associated with the greatest number of medication errors (MEs), whether harm is caused or not [5-7]. Recent systematic reviews of medication administration error (MAE) prevalence in healthcare settings found that they were common $[8,9]$, with one reporting an estimated median of $19.1 \%$ of 'total opportunities for error' in hospitals [8]. A significant proportion of MAEs are associated with actual or potentially harmful effects $[8,10]$.

The key to implementing a successful intervention that minimises MAEs is to understand how and why they occur. As nurses find themselves as the 'last link in the drug therapy chain' where an error can reach the patient [11], they have traditionally been blamed for errors [11, 12]. However, the reality is that the conditions within which the person responsible for the error works, as well as the strategic decisions of the organisation with whom they are employed, are often the key determinants of error [13, 14]. Frameworks for analysing medical error $[13,15]$ as well as taxonomies for recording MEs [16, 17] have been developed that recognise this distinction.

In order to determine the cause of error, one must appreciate the intentions of the person responsible for it [18]. To achieve this, one might ask directly, but care must be taken as MAE data generated in this way could be based on general nursing opinion rather than experiences of specific errors $[19,20]$, which may not reveal the true complexity of how individual MAEs arise. Another method would be to observe what causes errors as they happen without referring to the person directly involved with the error, whilst avoiding researcher opinion on causality [21]. Direct observation has been found to be the best available method for determining the prevalence of MAEs [22,23] and can identify potential error causes and associated factors [24], which the person making the error may not notice [25] or that retrospective methods of identification may not uncover [26].

Whilst some have summarised the literature on the causes of MAEs [27-33], to date there have been no attempts to systematically review studies concerning causes of MAEs in all patient types in hospital settings. Most of these reviews do not comment on the methodological quality of studies they found and include data generated from incident reports or general nursing opinion [27-32]. Therefore, a need exists to critically appraise this literature in order to ensure the causes of MAEs are determined based on a foundation of empirical (rather than surmised) data. The aim of this research was therefore to systematically review and appraise the empirical evidence available relating to the causes of MAEs in hospital settings.

\section{Literature Search Method}

\subsection{Search Strategy}

The following electronic databases were searched by RNK: MEDLINE, EMBASE, International Pharmaceutical Abstracts, Cumulative Index for Nursing and Allied Health Literature, PsycINFO, Health Management Information Consortium, Social Science Citation Index (all 1985-May 2013), British Nursing Index (1994-May 2013) and Applied Social Sciences Index and Abstracts (1987-May 2013).

Search terms used fell broadly into three groups: description of error [including error(s); medication error(s); incident report(s); near miss(es); drug error(s); treatment error(s); medication safety, drug safety, preventable adverse event(s), adverse event(s), medical error(s), clinical incident(s), adverse drug event(s), adverse health care event(s), health care error(s), medication incident(s)], variable of interest [cause(s); factor(s); reason(s); aetiology; etiology; causality; causalities; predictor(s); association(s)] and error type [including drug/medication/medicine administration(s); dose/drug/medicine/medication preparation(s); drug/medication/medicine delivery; omission(s); drug utilisation; commission(s); drug/medication/medicine supply; drug/medication/medicine handling; self medication; self administration]. Depending on database functionality, some terms underwent slight modification.

The reference lists of included studies and relevant review articles were hand searched to identify additional 
eligible studies. Study authors were not generally contacted for additional data. Once all database searches had been conducted, duplicate citations were identified and excluded using reference management software (EndNote $\mathrm{X} 3{ }^{\circledR}$ ) and manual title examination.

\subsection{Inclusion and Exclusion Criteria}

Studies that reported data on the causes of MAEs made in inpatient hospital settings published between 1985 and May 2013 were sought. Studies were included if they were published in English and identified causes in relation to specific errors or near misses that staff members either made themselves or were directly involved with. Relevant review articles were excluded, though their reference lists were hand searched for additional studies. Conference abstracts were excluded if they did not provide enough relevant data. Studies that reported on results based on simulation, or concerned with only one subtype of MAE, were excluded, as were studies reporting results obtained from incident or case reports as it could not be determined whether the person reporting the incident had been directly involved.

\subsection{Data Extraction Method}

The following details were extracted from all publications meeting the inclusion criteria: core details (including year of publication, first author, country of origin, study period), study background (including study type, setting, sampling strategy, drug administration route(s) studied, details of observers, subject details, definitions of administration error, error detection method(s), cause detection method(s), error framework categorisation if used), results (causes of MAEs), and additional information (including relevance of study to review aims). Data were extracted independently by RNK, JC and SDW; the authors met to resolve any differences in their results.

\subsection{Definitions}

An MAE was defined as 'a deviation from the prescriber's medication order as written on the patient's chart, manufacturers' instructions or relevant institutional policies' [8, 24, 34]. Ward-level medication preparation and dispensing errors were included, whilst prescribing and pharmacy dispensing errors were not.

Causes were defined as 'reasons reported to the researcher by the person directly involved with a specific administration error or near miss as being wholly or partly responsible for said error'. Direct observation could also provide data on causes of MAEs, provided it did not depend upon researcher opinion on causality.

\subsection{Data Analysis}

\subsubsection{Categorisation}

Extracted data on causes were aggregated and summarised according to theme, with Reason's model of accident causation $[18,35]$ used as a framework to categorise and present the data. Heterogeneity between studies meant that no attempt was made to quantify the frequency of MAE causes; such analyses would mislead readers, as a notion of frequency would be presented that may not reflect the true burden [21]. Instead, the number of papers reporting the presence of a particular cause were considered to identify (i) whether future research is required to explore this cause in more detail, and (ii) future potential targets for intervention to minimise MAEs.

Reason explains that systems such as healthcare have in place barriers or safeguards (e.g. double checking in healthcare) that protect a particular process or task from erroneous behaviour or subversion [13]. However, these defensive barriers can be weakened by decisions made during the design of the barriers and the wider systems in which they lie, and by actions or omissions of those people working on the front line. High-level design decisions may be flawed or simply a product of the constraints in which the managers function (e.g. financial, regulatory), and in turn shape the 'front line' working environment and can therefore make it error prone; together they form the 'latent' error pathway, and these working conditions are called 'error- or violationproducing conditions' [36] (see Fig. 1). The worker who carries out the task may also weaken the defensive barrier by making errors or not following procedure; these can be termed 'unsafe acts', and their effects are usually short lived when compared with managerial decisions and error-provoking conditions, which can lie dormant for many years until they are addressed $[13,35,36]$. Unsafe acts describe the acts or omissions of staff in the process of care and include skill-based errors such as 'slips' and 'lapses', as well as knowledge- and rule-based 'mistakes', and deliberate 'violations' of practice [35]. An unsafe act such as a nurse choosing the wrong drug to administer is more likely to be administered to a patient and result in an MAE if error- or violation-producing conditions in the working environment, as well as contributing high-level decisions, are present [13].

\subsubsection{Quality Assessment}

Quality assessment was integrated into the review process in two ways by RNK. First, by filtering out studies in which authors speculated as to the causes for MAEs, or where participants were asked to report on the causes of errors more generally, we ensured that only 'empirical' data based on specific error experiences were included. 


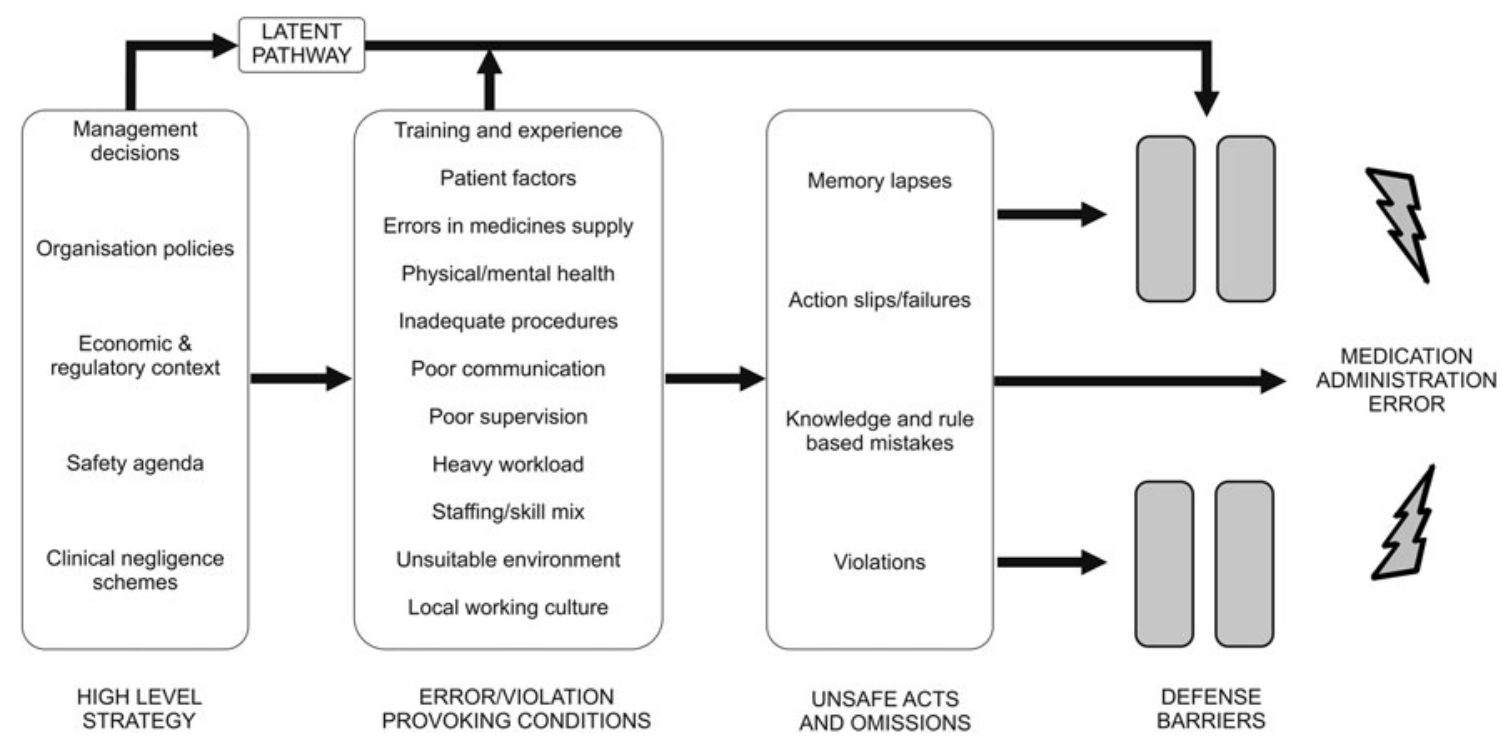

Fig. 1 Reason's model of accident causation as applied to medication administration errors in hospitals $[13,18,35,36]$

The second stage of quality assessment occurred after data extraction. Due to the heterogeneity of study designs, in-depth quality analysis was impractical; instead broad quality criteria were applied by RNK and considered relating to three main interests: the relevance of the study to the aims of this review; the method of sampling/sample size and finally the method of MAE causes data collection.

\section{Results}

\subsection{Search Process}

In all, 55 publications were eligible for inclusion. Three studies reported data from two countries [37-39] (and were considered six unique studies) and four [40-43] reported from the same data set as sister articles [34, 44, 45] (each group was considered one unique study) yielding 54 unique studies. A total of 21,799 articles were excluded at the title review stage as they were either duplications or were not related to medication safety topics. Abstracts were then reviewed and articles excluded if they were not thought to report on the causes of MEs. At the full-text examination stage, only studies focusing on the causes of MAEs in hospitals were included. A summary of the search process is shown in Fig. 2.

\subsection{Study Characteristics}

\subsubsection{Country of Origin}

Twelve $(12 / 54,22.2 \%)$ unique studies each originated from the UK [34, 37-40, 46-53] and the USA [6, 7, 38, 42, 43, 45, 54-61]. Six (11.1\%) originated from Australia
[62-67]; four (7.4\%) from South Africa [68-71]; three (5.6\%) each from New Zealand [72-74] and Germany [37, $39,41,44]$, and two each $(3.7 \%)$ from Canada [75, 76] and Malaysia [77, 78]. The remaining studies were each from separate countries: The Netherlands [79], Spain [80], India [81], Taiwan [82], Iran [83], Ethiopia [84], South Korea [85], China [86], Norway [87] and Turkey [88]. Study characteristics are summarised in Table 1.

\subsubsection{Study Setting and Patient Demographics}

A total of 20 studies were carried out in teaching hospitals $(37.0 \%)$ and 13 in general or unspecified hospitals $(24.1 \%)$. Tertiary care hospitals were the setting for four studies [56, 57, 69, 81] and an army medical centre for another [55]. Two studies were set in paediatrics hospitals $[65,74]$ and three did not report the institutions from which data originated [59, 68, 71]; however, because they reported MAE data from anaesthetists and inpatient settings, it was assumed that they had originated from hospital environments. Eleven studies were carried out in a range of clinical settings that included hospitals of various types.

Seven studies were conducted solely on paediatric units $(13.0 \%)$ [6, 55, 59, 65, 74, 78, 88]. Eleven were carried out using only adult patients (20.4\%). The remainder were either carried out with both adult and paediatric patients $(n=8,14.8 \%)[34,40,51,54,58,60,84,86,87]$ or the ages of patients were not specified $(n=28,51.9 \%)$.

Fourteen $(25.9 \%)$ studies were conducted on only one unit and $22(40.7 \%)$ on two or more units within each institution. Seven studies did not specify how many units per institution were observed but could have been numerous given the sampling strategy used $[42,43,45,52,54$, 


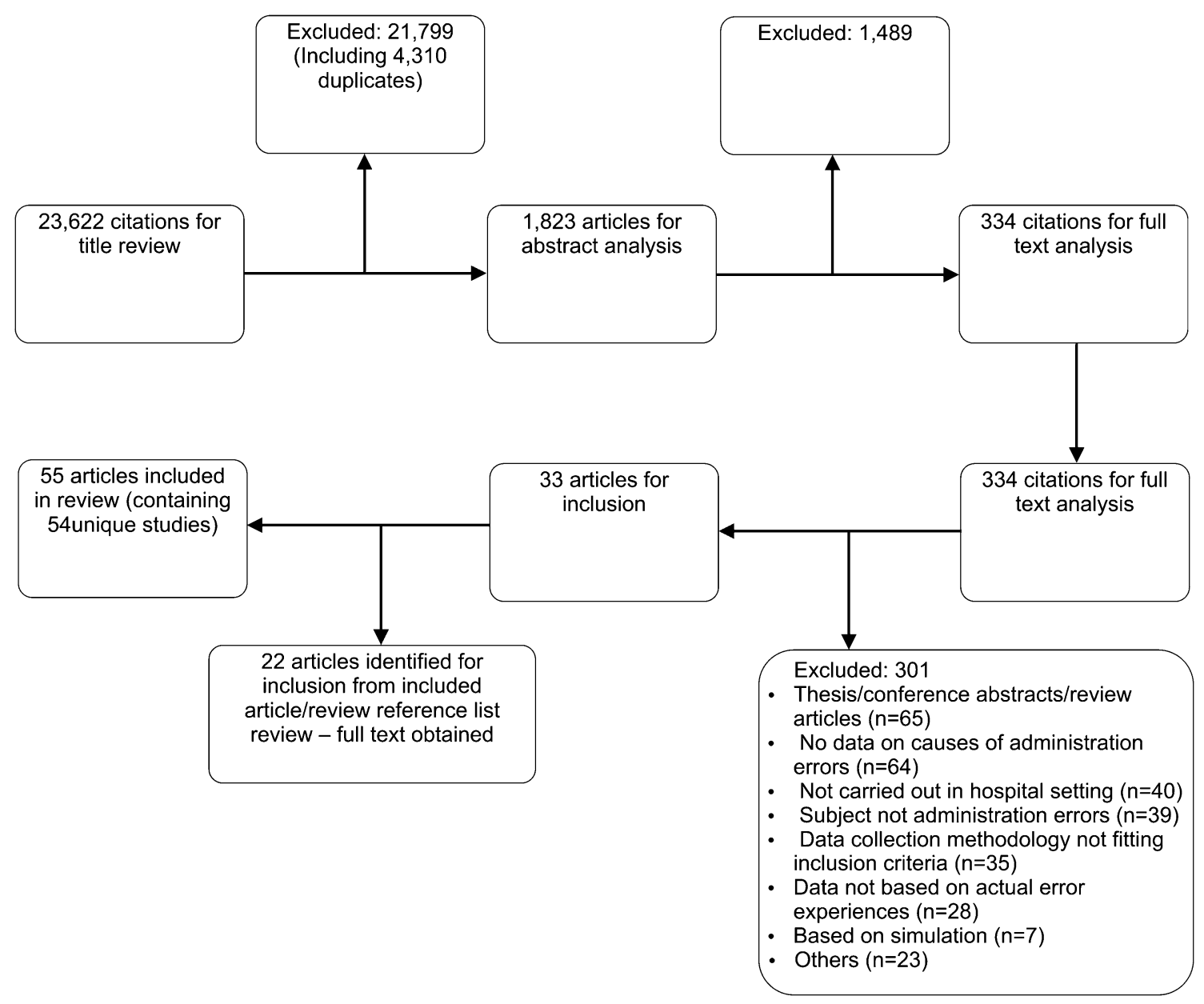

Fig. 2 Study identification and exclusion process

58, 63, 82, 85]. Nine studies carried out in anaesthesia could also have sourced data from more than one theatre per hospital based on their sampling techniques [68-72, 75, $81,86,87]$. Two studies did not state how many units were involved $[59,60]$.

\subsubsection{Study Design}

All studies utilising the direct observation method $(n=23$, $42.6 \%)$ identified MAEs prospectively, with some confirming the error once the medication administration round had finished $(n=8)$ and others during this activity $(n=10)$ (five studies did not specify when MAEs were confirmed). One study utilised observation with chart review [84] and another utilised interviews [67] for prospective and retrospective error identification. Those that did not utilise direct observation to identify MAEs employed a variety of error-detection methods; these included prospective self-reporting methods such as log books $(n=2,3.7 \%)[54,58]$, error (e.g. incident) reports $(n=3,5.6 \%)[53,56,57]$ and anaesthetic administration forms $(n=4)[69,72,86,87]$. Other prospective methods relied on other healthcare staff or researchers to identify errors through routine activity or chart review $[53,56,57$, 62]. Retrospective methods included interviews $(n=7)$ [51, 52, 59-61, 63, 66], questionnaires/surveys $(n=10)$ $[42,43,45,64,65,68,70,71,75,81,82,85]$ and focus groups $[73,74]$. Some of these studies described a validation process to confirm the presence of an error after the observation period $[6,7,56,57,77]$.

Besides the use of self-report methods to detect data $(n=16)$, MAEs were most often collected by pharmacists or pharmacy students $(n=19,35.2 \%)$ or nurses/nurse students $(n=9)$. Five studies did not specify who collected the data $[50,59,63,77,78]$ and five utilised combinations of various healthcare professionals $[53,56,57$, 80, 84].

Data on causes of MAEs were generally collected prospectively whilst directly observing staff activity on the unit(s) $(n=18)$. Some studies combined observation with chart review [84], informal conversations with staff $(n=2)$ [34, 40,41,44] or interviews $(n=4)$ [67, 77, 


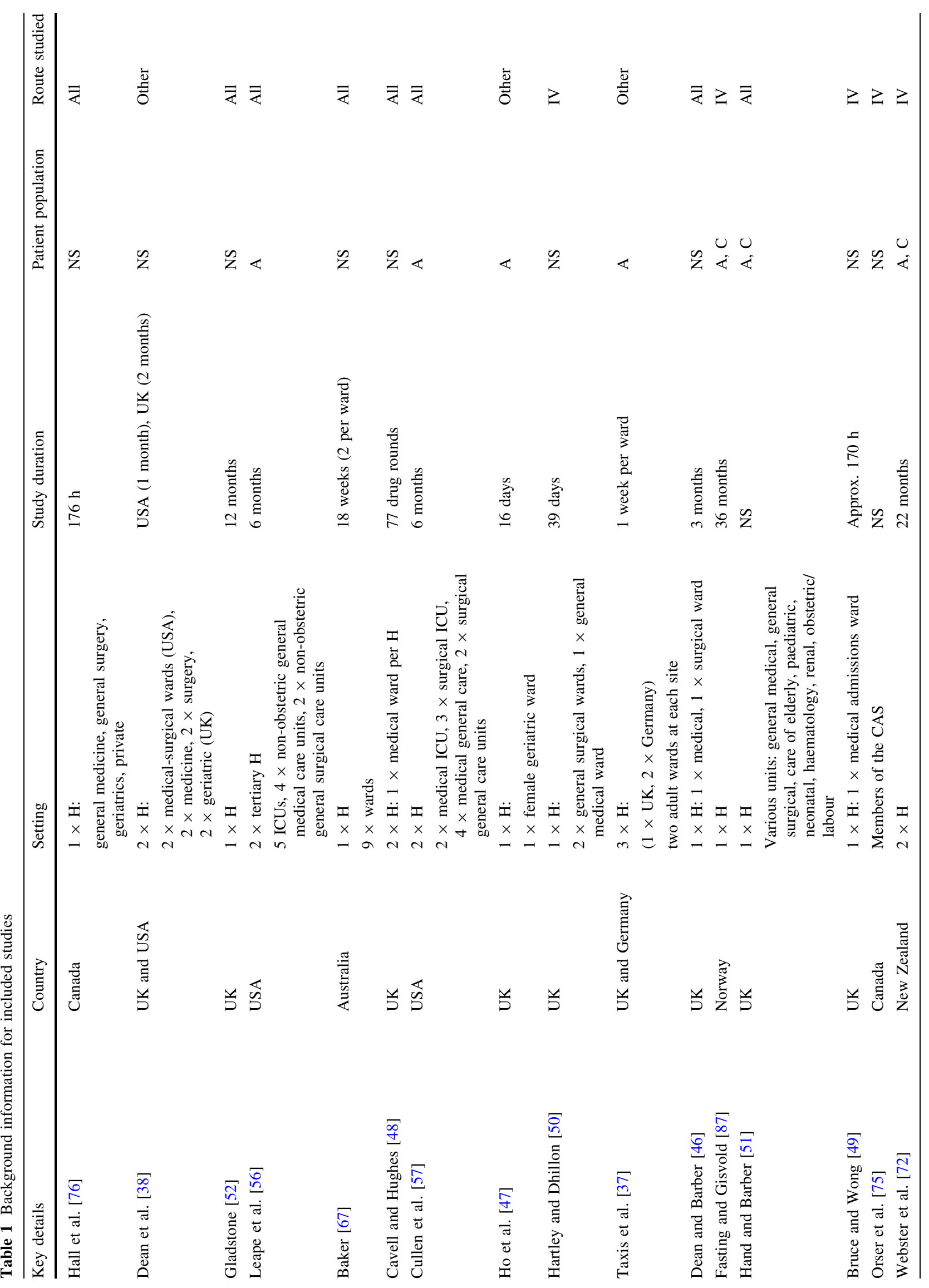




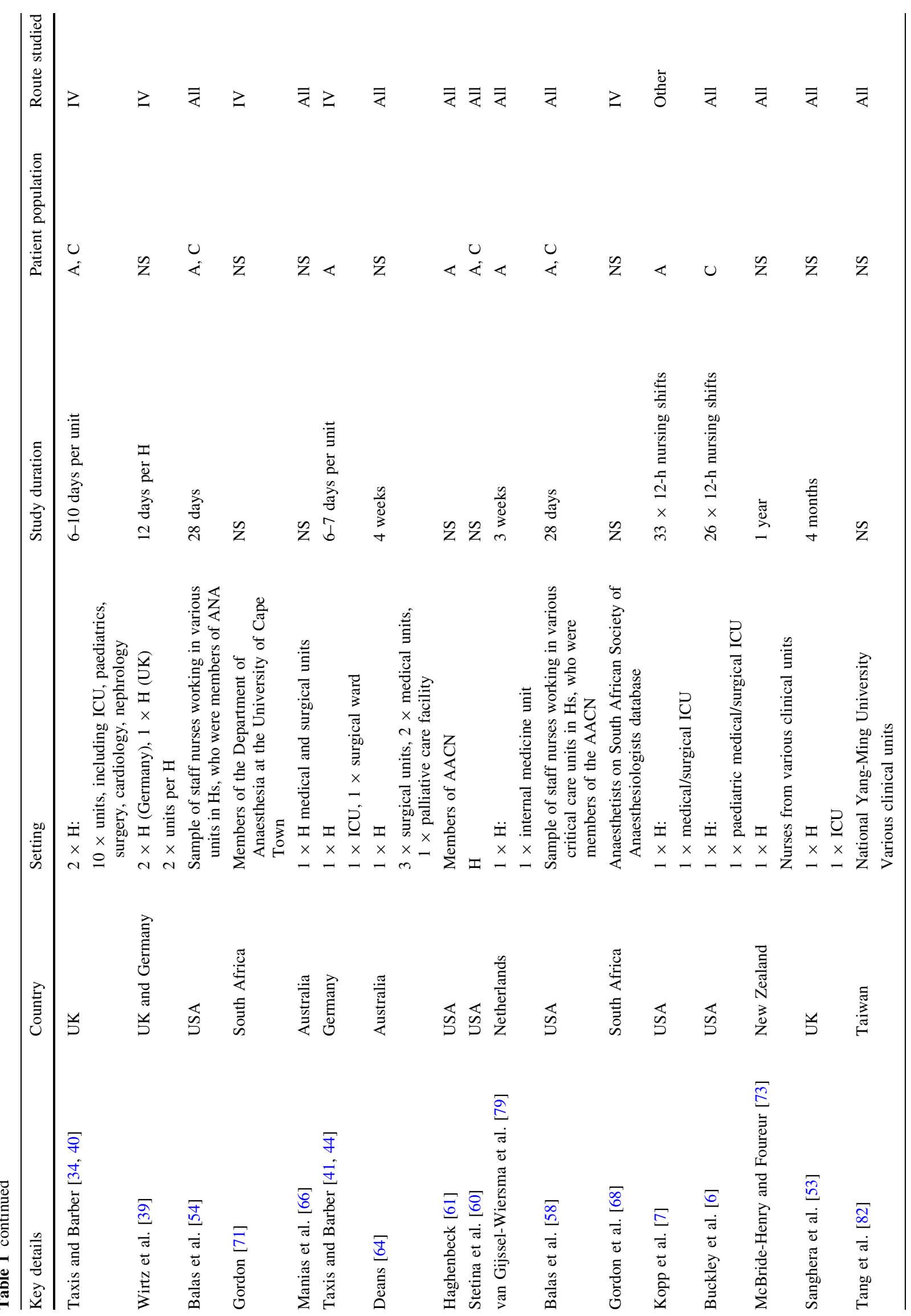




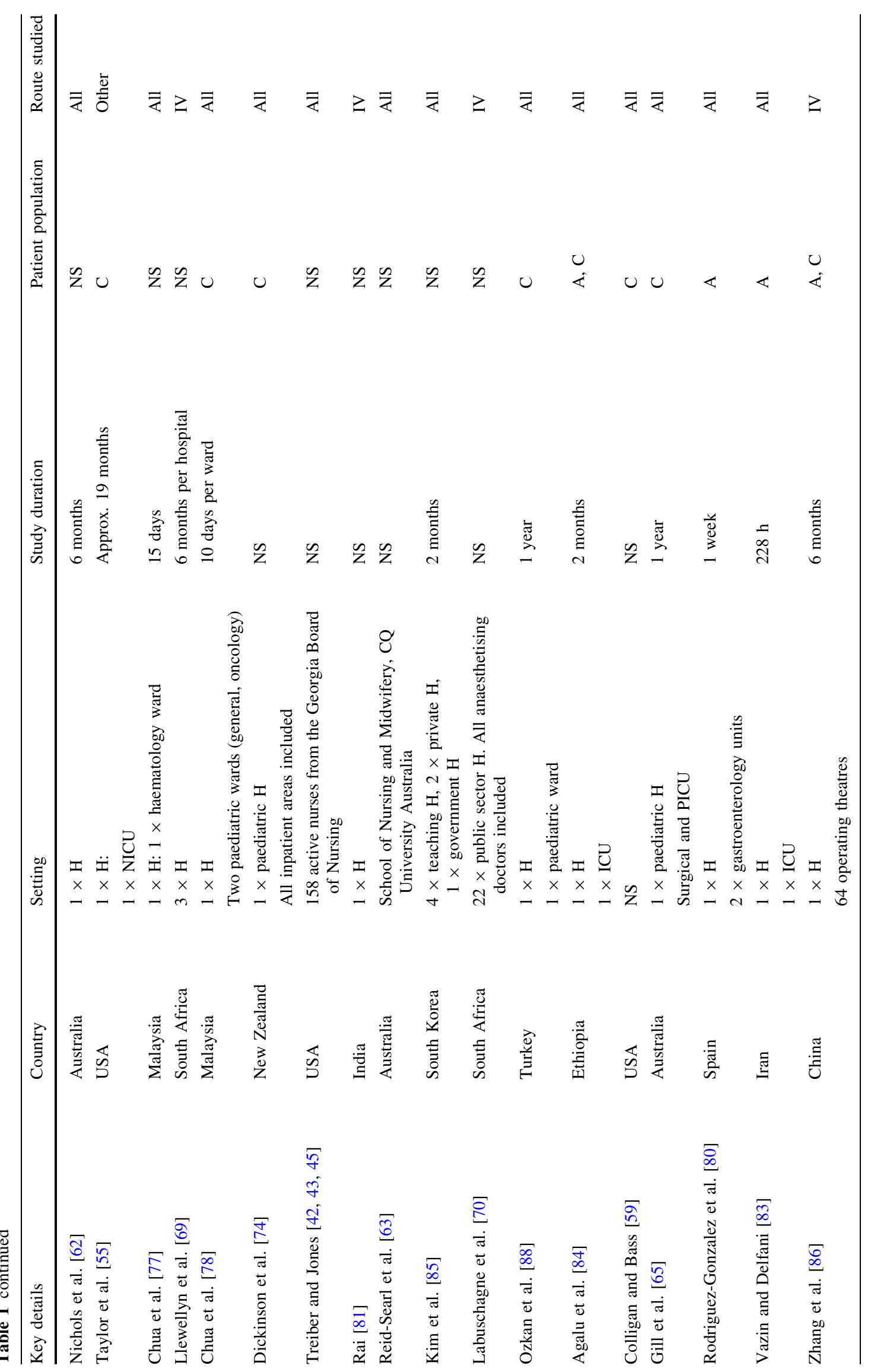




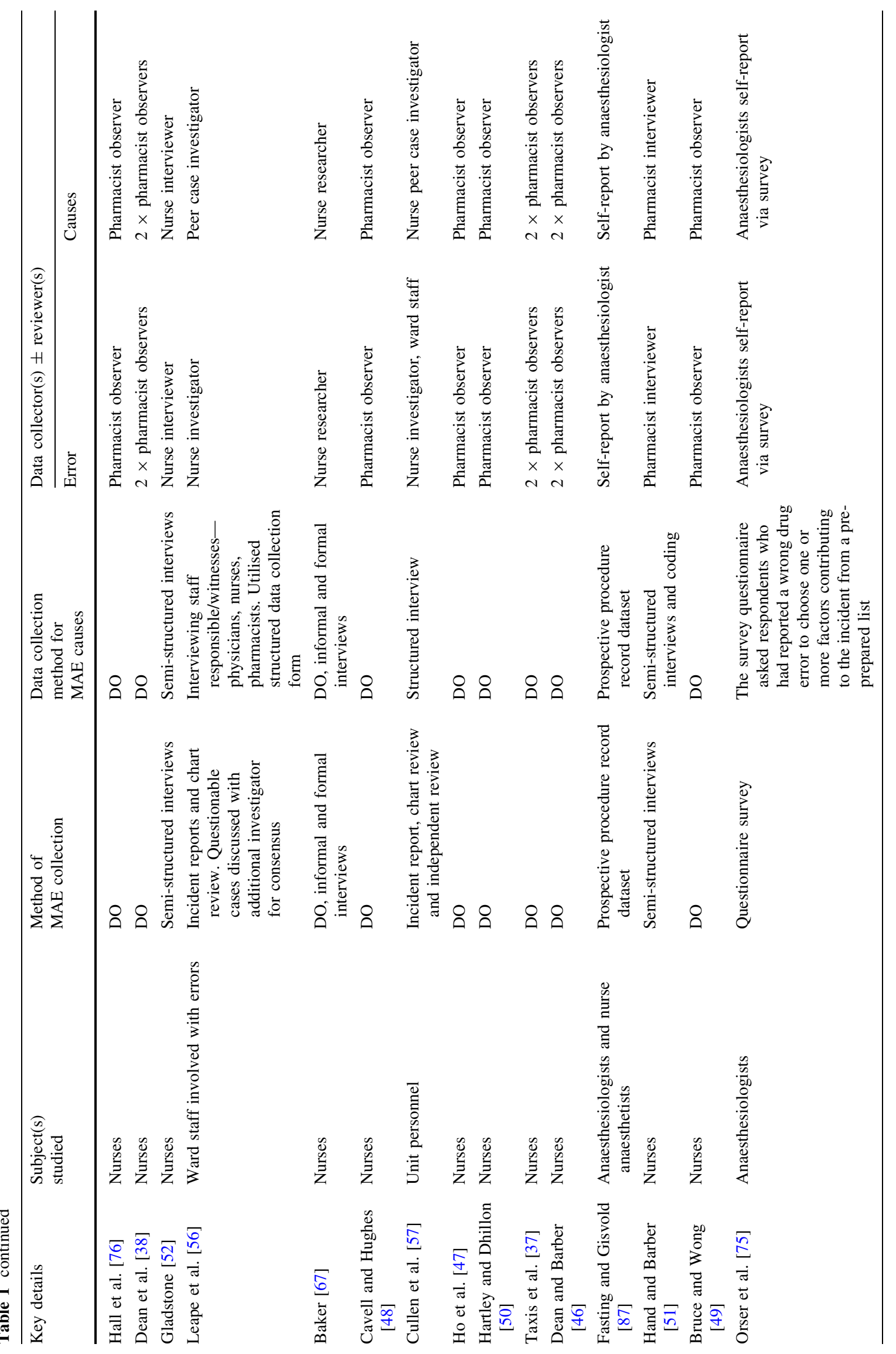




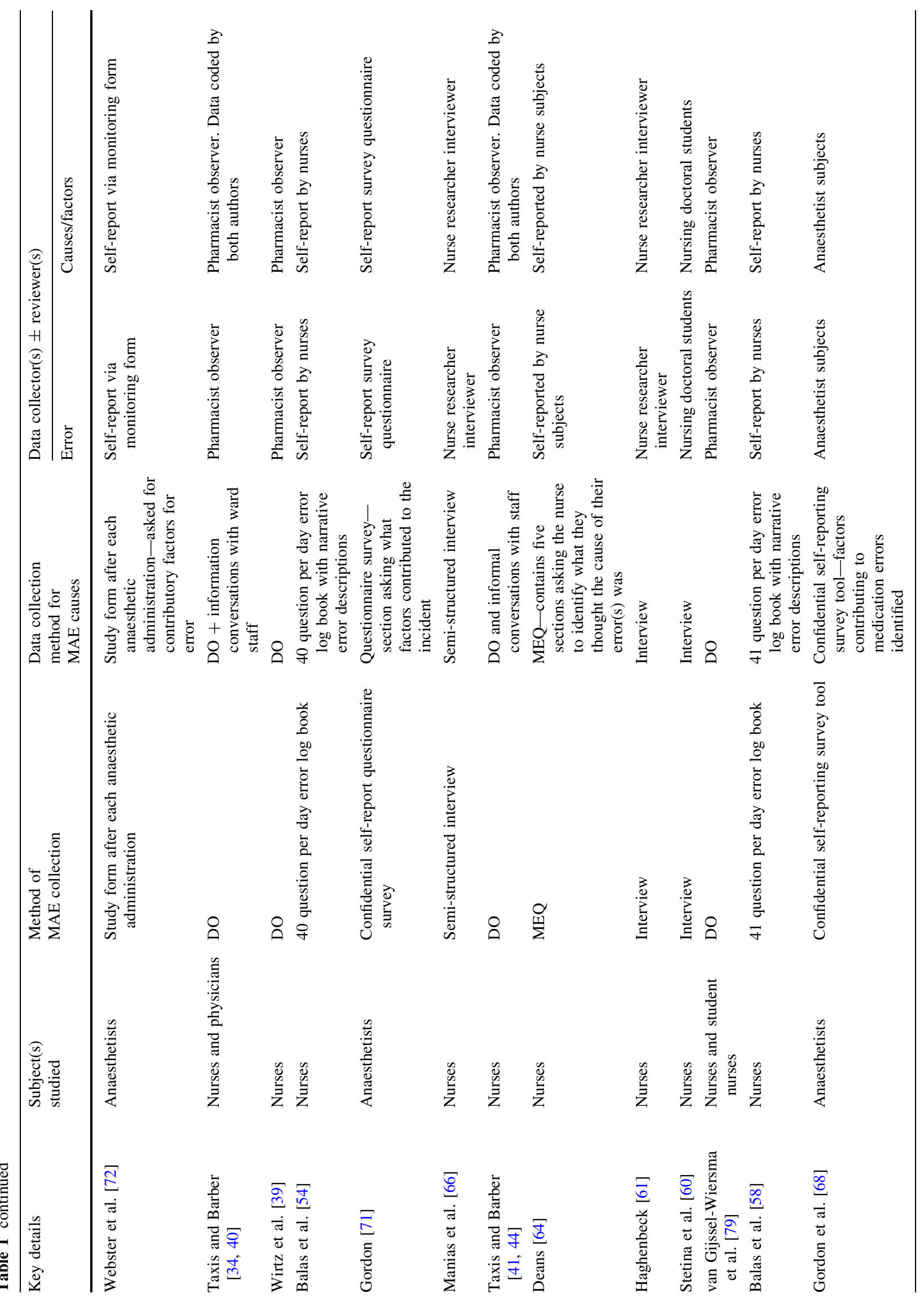




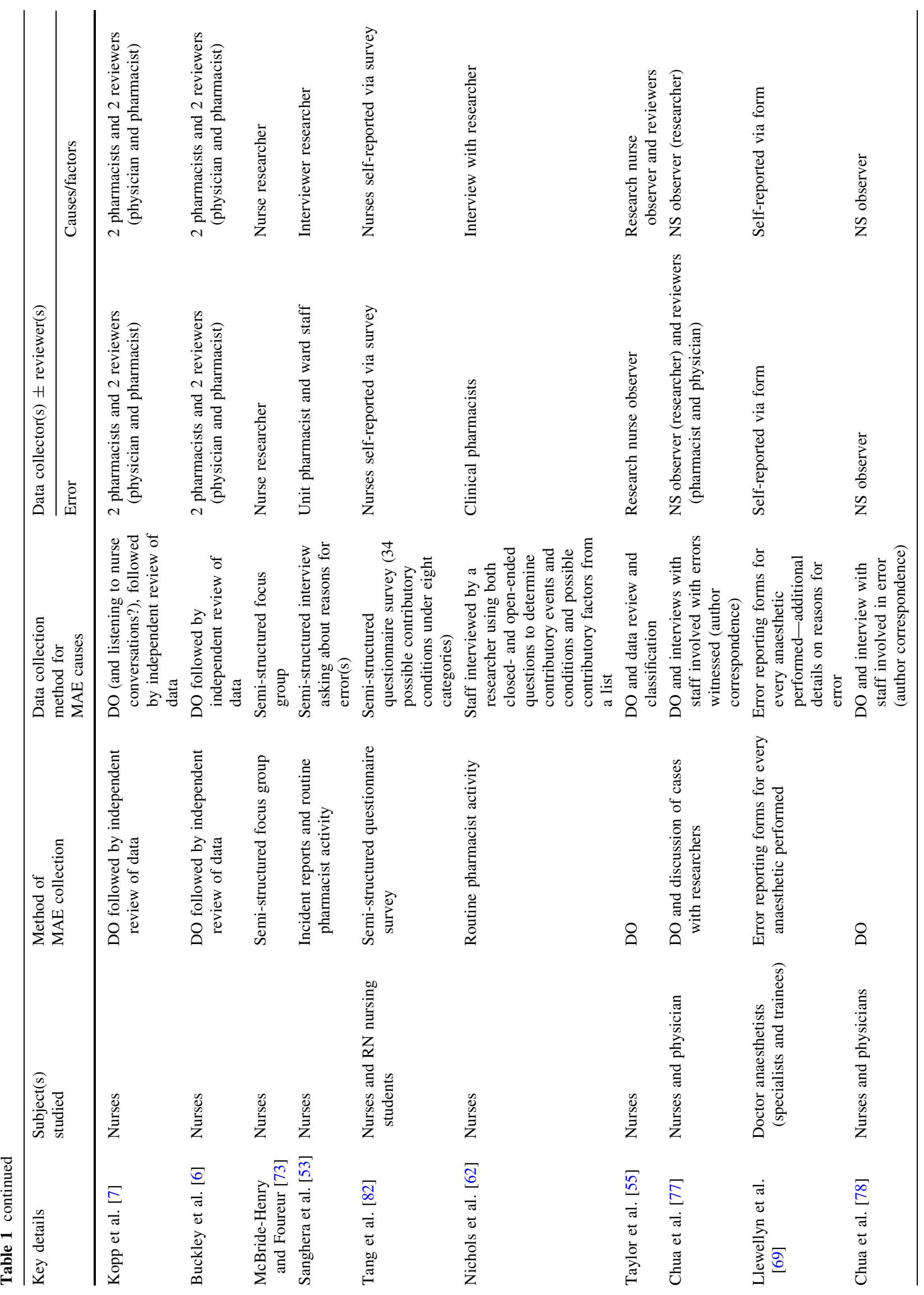




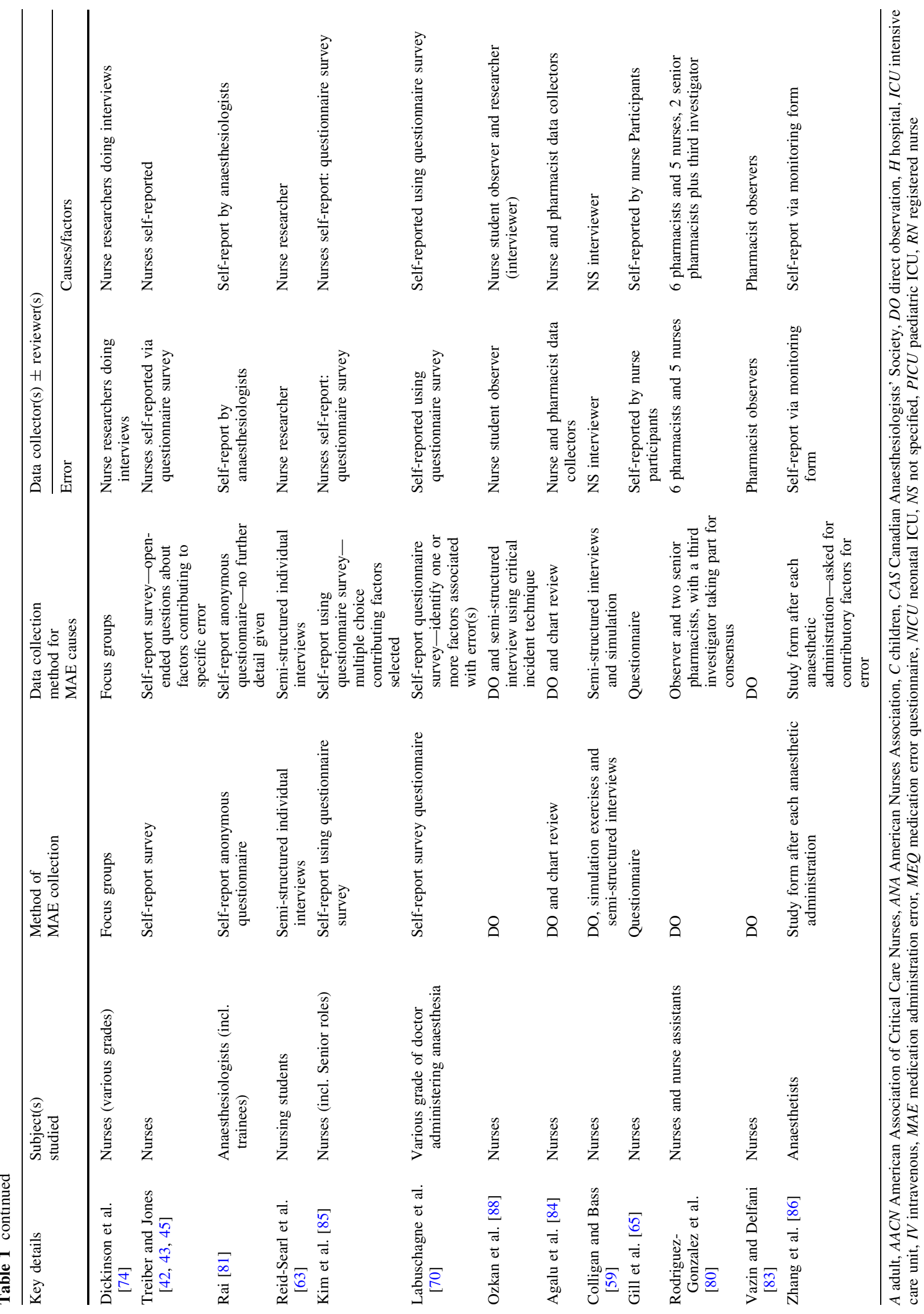


78, 88]. Self-report data was another common method to collect MAE causes; staff used daily log books $(n=2)$ [54, 58], incident forms for each anaesthetic procedure $(n=4) \quad[69,72,86,87]$ or surveys/questionnaires $(n=10)$ [42, 43, 45, 64, 65, 68, 70, 71, 75, 81, 82, 85]. Two studies utilised focus groups to collect these data $[65,73]$. Various types of interview were used by the remaining studies to report causes data $(n=11)$ [51-53, 56, 57, 59-63, 66]. Of those using survey methodology, two used open-ended questions to solicit data [42, 43, $45,85]$, three used a limited list of contributory factors from which participants could choose [75, 82, 85] and six did not state the type of questioning employed [64, 65, 68, 70, 71, 81]. Excluding self-reporting methods $(n=16)$, causes data collectors were most often pharmacists $(n=18)$, or nurses/nursing students $(n=7)$.

\subsubsection{Definition of a Medication Administration Error}

The definition of MAEs varied considerably between studies. Twenty-one (38.9\%) studies did not give a formal or working definition. Nine $(16.7 \%)$ studies used their own definition without referencing any established criteria. Of those who referenced criteria as either a complete formal definition or to supplement their own ( $n=24,44.4 \%)$, the most commonly used criteria used were those of Allan and Barker [24] and the American Society of Health System Pharmacists (ASHP) [89, 90].

\subsubsection{Route of Administration}

Fifteen studies $(27.8 \%)$ considered only the intravenous route of administration, whereas the majority $(n=32$, $59.3 \%$ ) studied all routes of administration. Seven studies observed MAEs via a varying number of different administration routes $[7,37,38,47,55]$. Nine studies in the intravenous group involved administration of medication used for anaesthesia [68-72, 75, 81, 86, 87].

\subsubsection{Staff Group}

The majority of studies investigated errors directly involving nurses ( $n=35,59.3 \%)$, student nurses [63] or both nurses and students [82] or nurses and nurse assistants [80]. Five studies involved nurses and medical staff [34, 39, 40, 77, 78] and eight studies obtained error data from various grades of physician responsible for administering anaesthetics [68-72, 75, 81, 86]. One study involved nurses and anaesthetists in theatres [87]. Two studies obtained data from various healthcare professionals who either made or were directly involved with nursing MAEs [56, 57].

\subsection{Quality Assessment}

Relevance of studies to review question. Overall, few studies were predominantly concerned with the causes of only MAEs $(n=6)$ [34, 40-45, 52, 63, 82]; most considered these issues after other major objectives such as the prevalence and nature of MAEs or more general experiences of healthcare staff when making errors. Of the latter group, examples included studies that focused on the causes of MEs made by a variety of healthcare professionals [53, 56, 57, 62], and investigations of nurse attitudes towards the defining or reporting of these errors and/ or their impact on professional practice [51, 53, 60, 64, 67]. Two asked participants to describe what factors influence their ability to carry out safe practice [73] or medicines management activities [66]. Sixteen studies (29.6\%) did not report any intention to study the causes of specific MAEs.

Sampling. The majority of studies provided insufficient detail of their sampling strategy to determine its nature. A minority $(n=5)$ reported random sampling of participants $[42,43,45,54,57,58,61]$, with only two describing the method of randomisation [42, 43, 45, 57]. Some publications reported sampling techniques where specific institutions or units were chosen; examples included wards with high error risk [47, 78] or wards chosen to reflect the patient population [76]. Another study recruited nurses from all units within their hospital as part of representative sampling [74]. Some studies interviewed staff based on errors previously identified by other staff members or the researchers [52, 53, 62, 77, 78, 88]. Two papers used the snowball sampling technique to recruit nurse participants [82, 85], two convenience sampling [39, 73] and nine selfreporting based studies sampled the entire population within specified limits (e.g. through registration databases) [68-72, 75, 81, 86, 87]. One study used patients admitted over a specific time period as the sample [56].

Reported sample size varied depending on the study method; $30(55.6 \%)$ reported the number of staff responsible for drug administration who took part in their study (e.g. nurses). Participant numbers varied between seven nurses in two studies [61, 62] and 720 anaesthetists in another [68]. Of the remaining studies, 21 provided details of the number of errors or the error rate, and three studies did not specify sample size [59, 60, 67].

Causes data collection method. Studies that utilised predominantly quantitative short answer surveys/questionnaires or direct observation methods alone were able to identify important causes of errors; these were generally limited in number (with a few exceptions [64, 82, 85]), did not contain more detail explaining why these causes arose and were not able to specify if multiple causes combined, as data were not generally related to individual errors. 
These studies listed causes in tables/text using very brief descriptors $[6,7,38,39,50,55,64,65,72,76,79-83,85]$. For direct observation, these results are not surprising considering that MAE causation data from observers that involved opinions or generalisations were excluded (many included such data $[6,7,38,48,50,55,76,80,83])$, leaving only those activities that were factual (e.g. delayed delivery of medication from pharmacy).

In contrast, interviews/conversations ( \pm direct observation), focus groups or self-reporting methods involving narrative free text responses generally provided a greater variety of MAE causes. Some demonstrated the link between administration errors/violations and their associated error-producing conditions using human error theory $[34,40,41,44,53,62,88]$. Additional verbatim quotes were used to confirm and expand upon data [34, 40-45, 51, $53,54,58,62,63,74,88]$, with some providing verbatim quotes of individual errors that demonstrated how multiple contributory factors combined to create MAEs [42, 43, 45, $54,58,62,63,73]$. However, not all of these studies provided much information specific to MAEs [53, 60-62, 66, 74], and some provided only brief tabulated/textual data of causes following interviews $[51,52,56,57,61]$ or direct observation with interviews [77, 78], much in the same way as survey/direct observation studies. Despite this, it is important to recognise that in some cases administration errors were not the sole ME of interest [53, 56, 57, 62], and many qualitative studies did not consider MAEs as their primary research topic.

One study used Reason's model of accident causation explicitly [53]. Seven studies used criteria that appeared to be based, at least in part, on elements of the systems approach to analysis of errors [6, 7, 55-57, 62, 83]. Three studies used elements of Reason's model along with other protocols for analysing adverse events [34, 40, 41, 44, 88]. Two studies used other referenced frameworks [64, 80]. Nine studies recorded a single reason or proximal cause for each reported MAE without offering further supplementary detail $[6,7,38,50,56,77,78,80]$. Five studies only investigated the causes of clinically significant MAEs [6, 7 , $56,57,62]$, with the remainder either basing their data on all types of MAEs or not distinguishing which type they considered (e.g. referred to only as MEs).

A number of causes/factors studies reported methods that had been tested in pilot/pre test phases $(n=15)[6,7$, $46,51,54,58,62,65,70,77,78,82,84,85,88]$. Others described run-in [79] or training phases [83] or based their method on earlier work [53, 86]. Only five studies determined causes of MAEs through triangulation of methods [34, 40, 41, 44, 77, 78, 88], which can be used to corroborate findings and, in the case of direct observation research when combined with interviews, bridge the gap between causes of errors that those observing practice cannot identify alone and those who make errors do not notice themselves [25, 40]. Despite this, few of these studies actually reported whether this actually was the case $[34,40,41,44]$.

\subsection{Reason's Model of Accident Causation}

The data from 54 studies presenting causes data were analysed thematically according to Reason's model and summarised in Table 2.

\subsubsection{Causes of MAEs}

3.4.1.1 Unsafe Acts Seven studies reported usable data matching Reason's description of active failures [34, 40, $41,44,53,56,57,62,88]$. The majority of studies identified primary causes of MAEs that could be attributed to the individual responsible for the error without using an established framework. These were broadly considered as either slips, lapses, mistakes or violations.

Slips and lapses. Slips and lapses were common, being identified by 29 studies (53.7\%). Misidentification of either medication or a patient were among the most frequently reported events considered as slips [54, 58, 68, 73, 76, 77, 81]. Misreading either a medication label/ product, prescription or other documentation was also common $[34,40,51,52,54,58,64,77,78]$. Staff confused look-a-like or sound-a-like medication names, patient names and medication packaging, which led to MEs [42, 43, 45, 56, 59, 63, 64, 69, 71, 72, 82, 85, 87]. Mental states such as lack of concentration, complacency and carelessness were also reported [51, 52, 78]. Most of the data was presented in tabular or list form in article texts; more detailed examples from qualitative interviews, focus groups or open-ended surveys were able to identify the cause(s) of their slips and lapses [34, 40-45, 53, 62, $63,73,88]$; these included nurses forgetting to sign a medication order or misreading labels due to being busy and/or distracted [42, 43, 45, 53, 88], failing to administer a drug or being careless due to heavy workload, poor staffing and/or being distracted [42, 43, 45, 73] and selecting the wrong medication due to pressure from others or busy/distracting environments [63].

Knowledge- and rule-based mistakes. Knowledge-based mistakes were less frequent $(n=16)$, with staff explaining that they did not know enough about the medication they were administering $[34,40,41,44,51,52,56,66,77,78$, 82], the infusion pump they were using [58] or the patient to whom they were administering it $[56,82]$. Rule-based mistakes were generally not observed [88].

Violations. Violations were reported by 14 studies, which were limited predominantly to data collection methods involving (at least in part) conversations with 
Table 2 Summary of medication administration error (MAE) causes reported by included studies

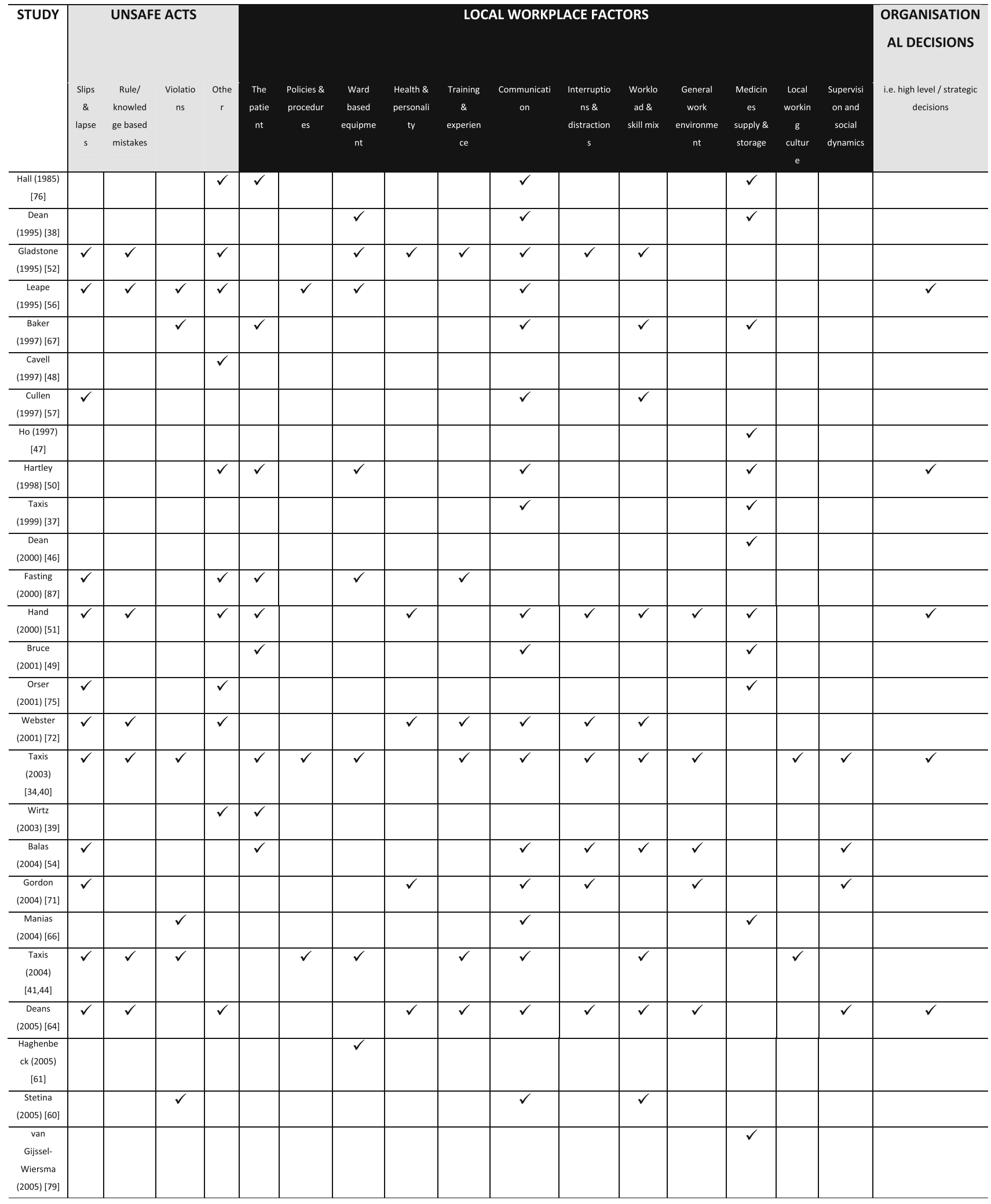


Table 2 continued

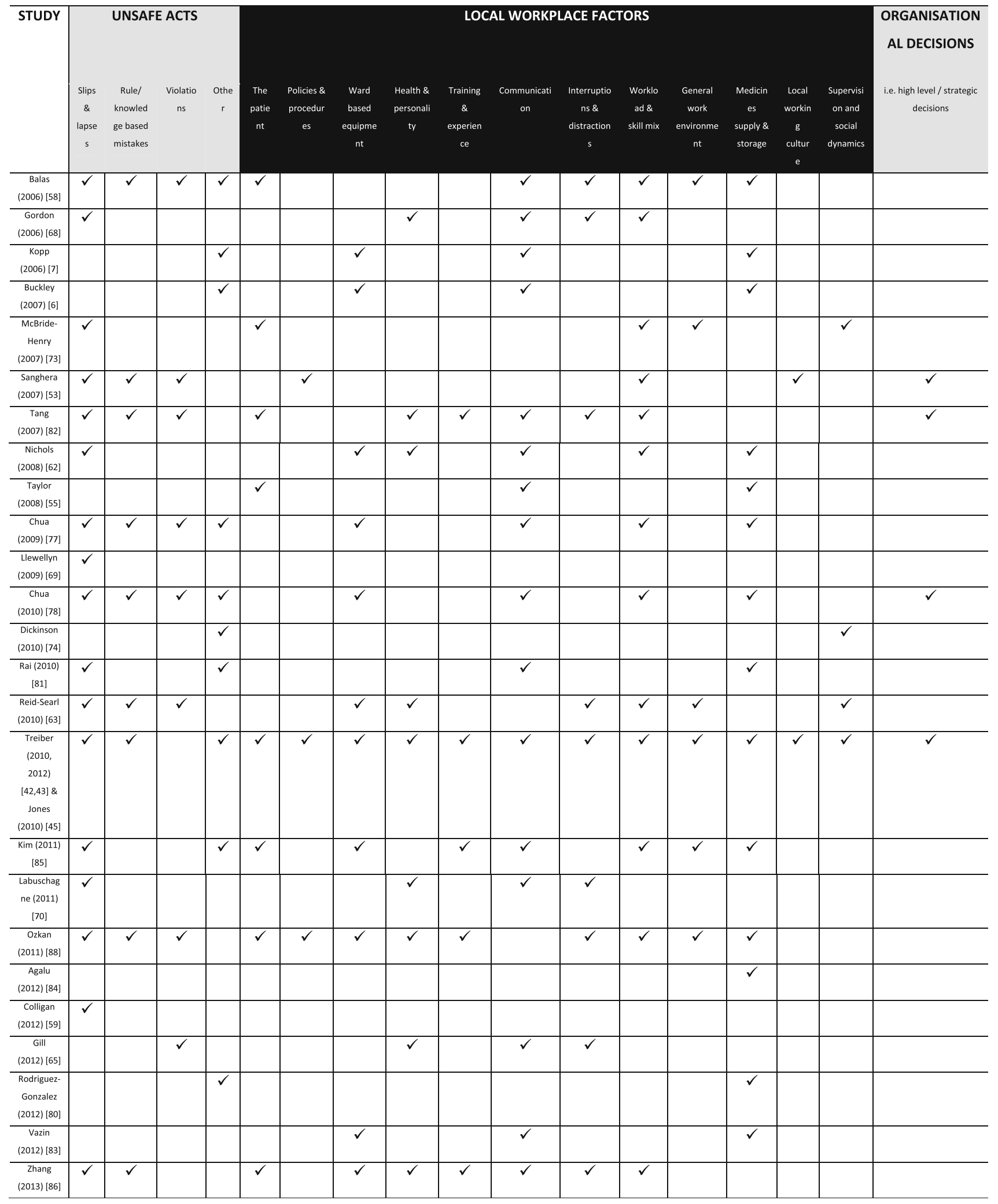

$\checkmark$ indicates that this type of MAE cause was described in the source document 
subjects to determine error causality. Where enough data were provided, situational violations (those arising due to necessity [e.g. poorly designed protocols, lack of staff]) were noted [53, 58, 67, 77, 78, 88]. Reasons for violations included trusting senior colleagues [63], patients requests [77], lack of access to suitable administration protocols [88], patient acuity [58, 88], acting in the patients' interests (e.g. to avoid harm or optimise treatment) [53, 60, 66], poor supervision/drug knowledge (associated with fast bolus intravenous administration) [34, 40], lack of staff (intentionally giving drugs early/late) [58, 67, 78] and common accepted practice (administering without a signed prescription) [53]. One nurse gave paracetamol 4 hourly instead of the prescribed 6-hourly regimen because they thought it would not be effective if given 6 hourly [66].

Other unsafe acts. Calculation errors [39, 48, 50, 52, 64, $74,77,78,80,87]$ and faulty checking activities [6, 7, 42, $43,45,56,63,72,75,77,78,80-82,86]$ were commonly reported. Difficulty with infusion equipment was also noted [42, 43, 45, 56, 58, 76, 83]. Other errors included not following instructions; insufficient evidence existed to determine whether these were deliberate acts (and hence violations) [51, 52, 77].

3.4.1.2 Latent Conditions Error- or violation-producing conditions describe the circumstances in which errors occur, and arise due to high-level managerial decisions. Multiple conditions can lead to one unsafe act [36].

The patient. A total of $17(31.5 \%)$ studies reported patient characteristics as causes of MAEs. Logistical problems associated with delivery of medication were most common and included lack of, difficulty with or delays waiting for intravenous access [34, 39, 40, 49, 50, 67, 68] (leading to wrong route [39], deterioration of medication [49], omission [50], wrong time [49] and compatibility errors [39]), and absent/sleeping patients during drug administration rounds [49, 51, 67, 76, 88]. Severity of patient illness (acuity) was reported by seven studies [42, 43, 45, 54, 55, 82, 86, 88]; some studies provided examples of resulting errors, which included wrong time or dose omission and, in many cases, the nurses were aware of their actions, which would constitute a violation [54, 58, 88]. Patient behaviour also led to MAEs through non-cooperation [34, 40, 51, 85], or prevented errors though knowledge of medications [73].

Policies and procedures. Problems with policies or procedures were reported on few occasions $(n=6)$. Examples included absence of a policy [41, 44, 56] and policies that were considered over-laborious [42, 43, 45], or generally unsuitable [34, 40, 41, 44, 88] (which led to wrong dose and time violations in one study [88]). Nurses reported that they had only basic information to help them safely mix and administer intravenous medications [41,
44]. Nurses were unclear about the role of the second checker in one study, which contributed to MAEs [53].

Ward-based equipment. Problems with equipment used to aid drug administration contributed to MAEs $(n=19)$. Insufficient equipment (computers [62] or gloves [78]) [88], malfunctioning equipment [86] and ambiguous equipment design (e.g. syringe driver, drug packaging) [34, $40,41,44,50]$ were reported; more general problems with drug charts included a lack of access [38, 50] and misplacement [50, 77], which combined with distractions and a noisy environment to lead to a wrong drug error in one interview study [63]. Example(s) of the nature of the infusion pump problems were given by a few studies, which reported that doses could either be administered incorrectly due to being un-calibrated [77] or malfunctioning (a nurse commented how she expected the pump to work because it was well tested) [61], or not be administered at all due to different pump properties [88] or pumps that were not connected [78].

Health and personality. Physical feelings of fatigue, tiredness/sleep deprivation, sickness and general discomfort amongst staff were reported as contributory factors to errors $(n=13)$ [42, 43, 45, 51, 52, 63-65, 68, 70-72, 82, 86, 88]. More detailed analysis of error accounts by one interview study revealed cases where physical exhaustion was caused by long hours and lack of breaks/food [63]. Staff member mental state at the time of error occurrence was also reported to lead to errors; stress [42, 43, 45, 51, 52, 62, 64], boredom [68], nervousness (with being busy and young) [42, 43, 45] and poor mood [82] were all found to be associated, though their origins were not stated. Personalityrelated causes were briefly reported as a lack of assertiveness/confidence [52] (including when challenging medical staff [51]), error perception [88] and conscientiousness [51].

Training and experience. Staff inexperience played a role in contributing to errors $(n=8)[34,40,42,43,45,52$, $72,82,86-88]$. This included being unfamiliar with the medication, environment, procedures or equipment, as well as being 'new' [42, 43, 45, 82, 87]. The feeling of being a newly qualified nurse in post was found in open-ended survey questions to be related to violation-type errors as nurses obeyed/trusted senior colleagues and felt pressure to complete their rounds on time, which led to them not performing their own safety checks [42, 43, 45]. Insufficient training and experience has strong links with knowledgeand rule-based mistakes [34, 40, 41, 44, 53, 88].

Inadequate training was also reported $(n=6)$, but few specifically mentioned training regarding the practicalities of preparing and administering medication [34, 40, 41, 44, 85]; one study reported that nurses felt their intravenous drug administration skills were not assessed appropriately [34, 40], which may have contributed to nurses learning these skills from each other on the ward [34, 40, 41, 44]. 
Communication. Difficulty with written communication featured prominently $(n=19)$, with two studies reporting that illegible [51, 64] and five unclear/messy [34, 40, 41, $44,55,77,82]$ prescriptions contributed to MAEs. Transcription errors were reported by some [37, 41-45, 50, 76, 83, 85], as were MAEs (e.g. omission and extra dose errors) apparently caused by others' documentation errors when writing prescriptions or administering medication $[42,43,45,50,60,65,67,77,78]$, with open-ended surveys and interviews relating one case to misinterpreting the roles of nursing students and their supervisors [42, 43, 45]. Studies commonly reported more general communication difficulties between healthcare staff or other services without specifying their nature $(n=15)$. Those using interviews/ conversations ( \pm direct observation) and open-ended survey methods reported instances where nurses/doctors failed to pass on information or successfully passed on incorrect information to their colleagues resulting in a drug administration delays $[66,67]$, drugs being given that should have been withheld [77] and incorrect doses being administered $[42,43,45,54]$. Problems with labelling were also frequently reported, though detail on their nature and relationship to other causes was missing [49, 68, 70-72, 81, 82, 86].

Supervision and social dynamics. Poor supervision by senior colleagues appears to have a role to play in MAE causation, manifesting as pressuring students to administer drugs more quickly, not supervising or assisting closely enough or giving unclear/incorrect instructions $(n=4)$ [34, 40, 42, 43, 45, 63, 86]. As discussed previously, specific error examples appear to link poor supervision to violation-type errors and the provoking conditions of inexperience, trusting colleagues and fatigue [34, 40, 42, $43,45,63]$, though supervision has also been linked to poor equipment and workload in one example [42, 43, 45]. Two studies reported apparent overconfidence in/from other nurses when either communicating instructions (as a cause of a wrong dose error) [42, 43, 45] or carrying out independent checks (a dose calculation error) [74]. Pressure from other staff members [42, 43, 45, 71], confronting and intimidating behaviour [64] and social isolation from colleagues also feature as causes [42, 43, 45]. There were examples of how proper supervision and communication could maintain patient safety, through co-workers identifying errors before they reached the patient [54, 63, 73].

Workload and skill mix. Heavy staff workload $(n=19)$ appeared an important contributor to MAEs, and includes end of shift/patient transfer pressures, patient load and multitasking [34, 40-45, 67]. Resulting errors included omissions [60] and violations [67, 88], though one study found that workload appeared not be a contributory factor (along with most other latent conditions) [57]. Workload was found to combine with distractions to lead to errors in intravenous administration [34, 40] and with patient acuity, inexperience or local working practice to lead to other errors [42, 43, 45, 62].

Skill mix of staff was identified by six studies, with two stating a lack of qualified staff $[34,40,51]$ and others that working with inexperienced or new staff members contributed to MAEs [64, 72]. Short staffing was reported by six studies as a cause of MAEs [42, 43, 45, 51, 64, 78, 82].

Distractions and interruptions. Sixteen studies found that interruptions/distractions were a cause of MAEs, though details of the nature of these distractions or their interplay with other contributory factors were rarely given. Of these studies, those using interviews/conversations ( \pm observation) or surveys with open-ended questions provided more descriptive data; examples of resulting errors/ near errors included wrong drug [58], wrong time [34, 40] and wrong dose calculations [88]. Distractions included ward rounds [34, 40] or face-to-face/telephone conversations with co-workers/patients [42, 43, 45, 63] and were often present with high workload and/or poor supervision [34, 40, 63].

General work environment. Eleven studies reported on the contribution of the general environment; specifically, noise [64], lighting [64, 71], emergencies [88], and busy $[34,40,42,43,45,54,58,73,85]$ or chaotic $[42,43,45$, 54] working environments were identified. Studies offering more detail through open-ended survey questions linked these factors to short staffing, workload, patient acuity and poor supervision [42, 43, 45].

Medicines supply and storage. Issues relating to medicines logistics were reported by 27 studies. A lack of ward stock led to omission/wrong time errors [37, 38, 47, 51, 78-80, 84]. Medication was misplaced or lost on the ward on occasions [47, 62, 75, 81]. In contrast, one study found no errors relating to medication unavailability [50]. The pharmacy department contributed to errors and violations in other cases, through delayed deliveries [50, 55, 66, 76, $85,88]$, incorrect dispensing [6, 38, 42, 43, 45, 51, 55, 58] and unavailable stock [58, 78, 79].

Local working culture. Nurses passed on bad practices (e.g. administering without a prescription) that led to errors in three studies [34, 40, 41, 44]. Levels of trust between colleagues [53] and working double shifts or not taking breaks (leading to exhaustion) [42, 43, 45] were additional causes. One nurse described how a wrong drug was selected for administration in a busy and chaotic theatre environment when she/he "relied on routine" [42, 43, 45].

Organisational (high-level) decisions. Included studies rarely reported organisational/high-level decisions as having a direct impact on error occurrence; feedback on errors was considered important by some interview or survey studies using narrative responses to minimise errors in future, and the importance of nurse input in the process was highlighted in one case [42, 43, 45]. Some described 
supervisory teams responding to errors poorly [63], that opportunities to learn from mistakes were limited [42, 43, $45,53]$ and how positive feedback about errors improved nursing practice $[63,64]$. More direct causal evidence cited a lack of hospital policy (when challenging other healthcare staff) or misguided policy (low nurse staffing) as causes of MAEs [56, 82], as well as decisions regarding logistical strategy generally revolving around clashes of other ward activities with medication administration [42, 43, 45, 50, 78]. Mix-ups involving medicines that look or sound alike may have roots beyond hospitals with the pharmaceutical industry [34, 40].

\section{Discussion}

Empirical evidence from this systematic review demonstrates that staff responsible for preparation and administration of medication are prone to making unsafe acts, but that their errors are heavily influenced by local working conditions. Key strengths of this systematic review are that it is the first to span hospital healthcare over the last 28 years and a critical approach has been used to assess study eligibility and quality.

There was little consistency between studies in terms of the method(s), settings and definitions they used. This may be in large part due to the varied purposes of the included studies. This inconsistency created differences in the types of data generated pertinent to the causes of MAEs and its level of detail; those studies that used certain data collection methods (interviews [with or without direct observation] and free-text self reporting), utilised error frameworks to present their data and/or focused on the causes of MAEs tended to provide a wider variety of MAE causes, details of their origins and whether or not they interacted with each other. Considering that only a small proportion of included studies predominantly sought to determine the causes of MAEs, and that only five of these used more qualitative methods [34, 40-45, 52, 56], the overall proportion of richer MAE causation data is small. Many studies reported data that included both potential unsafe acts and latent pathway causes without distinguishing between them $[6,7$, 48, 51, 52, 54, 56, 58, 64, 68, 71, 72, 77, 78, 80, 82].

In contrast with the above, studies that utilised more structured methods or used direct observation alone did not allow sufficient flexibility in questioning to allow the generation of the same level of causal detail [91], did not often provide information on how causal factors linked to specific MAEs (e.g. drug involved) or in the case of observation alone could not explore thought processes that underpinned actions as staff were not interviewed [24, 25, 40]. Given the evidence that prescribing errors can arise as a result of multiple, interconnecting factors [92], the result is that few studies without most of the methodological restrictions described above are able to generate error accounts that reflect this phenomena [34, 40-45, 54, 58, 63, 88]. Of these few, most do not provide break-down at the individual error level.

In light of the strong influence of data collection method on our findings, we did not attempt to infer meaning from the frequency of causes reported across included papers, as this may be misleading. One example is 'medicines supply and storage' issues, which feature strongly $(n=27)$, largely due to data derived from direct observation alone ( $\sim 50 \%$ of studies) - a method that identified a limited number of MAE causes restricted to the latent pathway.

More general limitations of self-reporting methods such as log books, administration reports, interviews and questionnaires include being subject to social desirability bias [93], potentially leading to modified reporting of MAEs [21]. Though direct observation may be susceptible to biased observer inference and the 'Hawthorne effect', whereby behaviour is modified in the presence of the observer [25], the effect of these phenomena can either be controlled through appropriate training for the former [24] or appears to be limited for the latter [26]. Alternative methods to identify potential causes of MAEs include prospective risk analysis methods such as failure mode effects analysis [94], but they have their own limitations [95] and may use general options as well as specific error accounts in their design.

Despite the above challenges associated with the body of literature, important causes of MAEs were identified. Slips and lapses were the most commonly identified unsafe acts, followed by violations and knowledge-based mistakes. Misidentification or misinterpretation of prescriptions or patients appeared to occur frequently but their origins were not well described. Though sparse, more detailed information showed that slips and lapses were related to distractions, high workload and poor staffing, and that confusion with drug names/packages may arise from planning of medication locations and pharmaceutical manufacturer designs [34, 40, 48, 64, 68, 69, 71, 75, 81]. Bar code-assisted administration has been associated with a decrease in some types of MAEs but increases in risk for others [96], as well as introducing novel errors in the form of 'work-arounds' [97]. The problems with technology identified in this review further emphasise the need to ensure its careful design and implementation.

Inadequate knowledge of medication appears to be a well recognised contributor to MAEs [27, 30, 32] and may extend back to inadequate teaching of clinical pharmacology [27]. Nurses' knowledge of 'high-risk' medication groups, such as cardiovascular and electrolytes may be limited [98], which is important given their association with MAEs [8]. 
Although problems with checking around medication administration were frequently reported, little detail was provided as to their nature. To date there is scant evidence to support the notion that double checking reduces the MAE rate [99]. Although failure in following policy/procedures may be a common contributing factor to MAEs [27, 28, 30, $32]$, it has been suggested that repeated identity checking undermines the nurse-patient relationship [100]. The small number of studies providing insight into the origins of violations suggests that their origins may lie in staff relationships, patient interactions, general workload and institutional policies and procedures. Recent work suggests that violations of medication administration protocols may be more likely in certain circumstances [101] and that they arise depending on the nurses workload, familiarity with the drug and patient, and local working culture [65].

The volume and range of written communication errors described indicates that they are a widespread, persistent problem in secondary care. Unsuitable prescriptions featured prominently, which suggests that quality administration is dependent on other healthcare professionals performing their roles appropriately. This is further emphasised from more detailed accounts of errors resulting from incorrect provision of information or documentation by colleagues, though further research exploring their origins are required, as so far these appear limited to a misunderstanding of the student/supervisor relationship. Transcribing errors were reported but appear to occur mainly in countries where nurses were expected to transcribe physician orders [37, 38, 41, 44]. The presence of pharmacy dispensing errors and more specifically ward stock/supply problems on the MAE causal pathway highlights additional communication challenges. Electronic prescribing and printed prescriptions may improve the prescribing process $[14,102]$, though mixed changes in some ME subtypes have been noted [48, 79, 102]. A closed loop electronic prescribing, dispensing and administration system may help confront some of the problems between different healthcare professionals identified above [103].

High workload has links to poor staffing, which in turn are both therefore related to organisational decisions regarding recruitment, though few studies made this association [42, $43,45,82]$. Most data on workload comprise brief descriptions with limited evidence suggesting aetiology and whether combinations with other causes such as interruptions/ distractions and inexperience lead to errors; more evidence is therefore required to explore the role of workload in MAE causation. Studies have linked nurse staffing levels to negative patient outcomes, including MEs [104], but this relationship is complex, and further study is required to understand more clearly the role MAEs have to play.

Distractions and interruptions disrupt concentration and attention, which can lead to loss in patient focus [105] and subsequently incorrect actions or omissions that result in errors [18]. Direct observation-based studies demonstrate that interruptions increase the occurrence of MAEs across different healthcare settings [106-108]. Despite highlighting interruptions as a common problem, this review found examples of their nature were limited to conversations, phone calls and patient acuity (some described delaying/ missing dose administration when they had to deal with a sick patient). More focused work has characterised the nature of interruptions $[59,106]$, and recommendations for minimisation could involve training nurses to prioritise multiple requests and targeting those interruptions that are preventable $[59,109]$. No studies have evaluated the effect of an intervention designed to reduce interruptions and MAEs using a direct observation MAE-detection method.

Despite poor physical and mental condition being a common contributor to MAEs, few examples of the aetiology or interaction of these conditions were provided, with the exception of exhaustion, which has been linked to working hours and lack of shift breaks. Working hours have been linked to increased nursing errors of various kinds (including MAEs) [104]. With a continuing drive to implement healthcare technology to improve patient safety [110], it is of vital importance that issues relating to proper maintenance, access and ease-of-use, identified as causes of MAEs in this review, are addressed in their design and implementation. When considered with the prominence of medicines supply issues, medication administration can be viewed as the culmination of multiple high-risk processes that complicate nursing practice and place patients in potential danger.

Organisational safety culture refers to individual and group values, attitudes, competencies and behavioural patterns that decide the organisation's safety programmes [111]. The nature and influence of this culture on MAEs remains relatively unexplored, as do wider organisational strategic decisions, where findings were generally limited to indirect associations involving logistical problems when scheduling medication administration with other ward activities and the effects of error feedback mechanisms. This may be because evidence has predominantly originated from 'front-line' staff rather than organisational leaders and managers.

Compared with studies involving nurses, research in anaesthesia utilised more restrictive self-reporting methods exclusively, resulting in the identification of few contributory factors of unknown aetiology. Of the remaining studies considering only the intravenous administration route, the focus of all but two [34, 40, 41, 44] was not predominantly on the causes of MAEs. Although these provided useful data, they did not utilise interviews with those who made the errors, which the authors themselves state may limit the identification of more personal error causes. 


\subsection{Limitations of Review}

By excluding non-English publications, the potential for publication bias may exist when focusing on the causes of MAEs. As we gathered evidence from both qualitative and quantitative studies, we were only able to compare study quality/relevance at a limited level, though our appraisal process was able to identify important areas for further exploratory research and potential interventions to minimise MAEs in hospitals.

\section{Conclusions}

This systematic review has demonstrated that MAEs have multiple causes, including both unsafe acts and error-/ violation-provoking conditions, and that a limited number of these conditions may combine in unique situations to result in error. However, due to a number of reasons, this intricate pathway has thus far only been superficially explored. There is a lack of consistency in approaching MAE causation research with regards to the methods used and whether error causation frameworks are utilised to analyse results. Given that a number of existing (and sometimes complex) interventions have shown that their results only partially address the MAE challenge, and in some cases create novel error opportunities, it is recommended that further research with a strong theoretical focus be undertaken to investigate the nature and complexity of causes behind MAEs, with a particular emphasis on which interventions may result in substantial, long-lasting improvements in patient safety.

Acknowledgments Richard N Keers gratefully acknowledges funding to support his $\mathrm{PhD}$ training provided by the University of Manchester and University Hospital of South Manchester NHS Foundation Trust (UK), as well as the advice offered by Dr Denham Phipps and Professors Bryony Dean Franklin and Charles Vincent.

Conflict of interest No conflicts of interest are declared by Richard N Keers, Steven D Williams, Jonathan Cooke and Darren M Ashcroft.

Open Access This article is distributed under the terms of the Creative Commons Attribution Noncommercial License which permits any noncommercial use, distribution, and reproduction in any medium, provided the original author(s) and the source are credited.

\section{References}

1. Leape LL. Errors in medicine. Clin Chim Acta. 2009;404(1):2-5.

2. de Vries EN, Ramrattan MA, Smorenburg SM, et al. The incidence and nature of in-hospital adverse events: a systematic review. Qual Saf Health Care. 2008;17(3):216-23.

3. Kongkaew C, Hann M, Mandal J, et al. Risk factors for hospital admissions associated with adverse drug events. Pharmacotherapy. Epub 2013 May 17. doi:10.1002/phar.1287.
4. Hug BL, Keohane C, Seger DL, et al. The costs of adverse drug events in community hospitals. Jt Comm J Qual Patient Saf. 2012;38(3):120-6.

5. Bates DW, Boyle DL, Vander Vliet MB, et al. Relationship between medication errors and adverse drug events. J Gen Intern Med. 1995;10(4):199-205.

6. Buckley MS, Erstad BL, Kopp BJ, et al. Direct observation approach for detecting medication errors and adverse drug events in a pediatric intensive care unit. Pediatr Crit Care Med. 2007;8(2):145-52.

7. Kopp BJ, Erstad BL, Allen ME, et al. Medication errors and adverse drug events in an intensive care unit: direct observation approach for detection. Crit Care Med. 2006;34(2):415-25.

8. Keers RN, Williams SD, Cooke J, et al. Prevalence and nature of medication administration errors in healthcare settings: a systematic review of direct observational evidence. Ann Pharmacother. 2013;47(2):237-56.

9. McLeod MC, Barber N, Franklin BD. Methodological variations and their effects on reported medication administration error rates. BMJ Qual Saf. 2013;22(4):278-89.

10. Kale A, Keohane CA, Maviglia S, et al. Adverse drug events caused by serious medication administration errors. BMJ Qual Saf. 2012;21(11):933-8.

11. Pepper GA. Errors in drug administration by nurses. Am J Health Syst Pharm. 1995;52(4):390-5.

12. Armitage G. Human error theory: relevance to nurse management. J Nurs Manag. 2009;17(2):193-202.

13. Reason J. Human error: models and management. BMJ. 2000;320(7237):768-70.

14. Kohn LT, Corrigan JM, Donaldson MS, editors. To err is human: building a safer health system. Washington, DC: National Academy Press; 1999.

15. Vincent C, Taylor-Adams S, Stanhope N. Framework for analysing risk and safety in clinical medicine. BMJ. 1998;316(7138):1154-7.

16. National Coordinating Council for Medication Error Reporting and Prevention (NCC MERP). NCC MERP taxonomy of medication errors. 1998. Available from URL: http://www.nccmerp. org/pdf/taxo2001-07-31.pdf. Accessed: 2013 July 2.

17. National Reporting and Learning Service. RLS Help System. Available from URL: https://www.eforms.nrls.nhs.uk/ staffreport/help/ALL/eForm_Help.htm. Accessed 2013 July 2.

18. Reason J. Human error. Cambridge: Cambridge University Press; 1990.

19. Mayo AM, Duncan D. Nurse perceptions of medication errors: what we need to know for patient safety. J Nurs Care Qual 2004; 19(3):209-17.

20. Ulanimo VM, O'Leary-Kelley C, Connolly PM. Nurses' perceptions of causes of medication errors and barriers to reporting. J Nurs Care Qual. 2007; 22(1):28-33.

21. Tully MP, Ashcroft DM, Dornan T, et al. The causes of and factors associated with prescribing errors in hospital inpatients: a systematic review. Drug Saf. 2009;32(10):819-36.

22. Flynn EA, Barker KN, Pepper GA, et al. Comparison of methods for detecting medication errors in 36 hospitals and skillednursing facilities. Am J Health Syst Pharm. 2002;59(5):436-46.

23. Meyer-Massetti C, Cheng CM, Schwappach DLB, et al. Systematic review of medication safety assessment methods. Am J Health Syst Pharm. 2011;68(3):227-40.

24. Allan EL, Barker KN. Fundamentals of medication error research. Am J Hosp Pharm. 1990;47(3):555-71.

25. Barker KN. Data collection techniques: observation. Am J Hosp Pharm. 1980;37(9):1235-43.

26. Dean B, Barber N. Validity and reliability of observational methods for studying medication administration errors. Am J Health Syst Pharm. 2001;58(1):54-9. 
27. Brady AM, Malone AM, Fleming S. A literature review of the individual and systems factors that contribute to medication errors in nursing practice. J Nurs Manag. 2009;17(6):679-97.

28. Armitage G, Knapman H. Adverse events in drug administration: a literature review. J Nurs Manag. 2003;11(2):130-40.

29. Fry MM, Dacey C. Factors contributing to incidents in medicine administration. Part 1. Br J Nurs. 2007;16(9):556-8.

30. McBride-Henry K, Foureur M. Medication administration errors: understanding the issues. Aust J Adv Nurs. 2006; 23(3):33-41.

31. Carlton G, Blegen MA. Medication-related errors: a literature review of incidence and antecedents. Annu Rev Nurs Res. 2006;24:19-38.

32. O'Shea E. Factors contributing to medication errors: a literature review. J Clin Nurs. 1999;8(5):496-504.

33. Gonzales K. Medication administration errors and the pediatric population: a systematic search of the literature. J Pediatr Nurs. 2010;25(6):555-65.

34. Taxis K, Barber N. Ethnographic study of incidence and severity of intravenous drug errors. BMJ. 2003;326(7391):684.

35. Reason J. Managing the risks of organizational accidents. Aldershot: Ashgate Publishing Limited; 1997.

36. Reason J. Understanding adverse events: human factors. Qual Health Care. 1995;4(2):80-9.

37. Taxis K, Dean B, Barber N. Hospital drug distribution systems in the UK and Germany-a study of medication errors. Pharm World Sci. 1999;21(1):25-31.

38. Dean BS, Allan EL, Barber ND, et al. Comparison of medication errors in an American and a British hospital. Am J Health Syst Pharm. 1995;52(22):2543-9.

39. Wirtz V, Taxis K, Barber ND. An observational study of intravenous medication errors in the United Kingdom and in Germany. Pharm World Sci. 2003;25(3):104-11.

40. Taxis K, Barber N. Causes of intravenous medication errors: an ethnographic study. Qual Saf Health Care. 2003;12(5):343-7.

41. Taxis K, Barber N. Causes of intravenous medication errorsobservation of nurses in a German hospital. J Public Health. 2004;12(2):132-8.

42. Treiber LA, Jones JH. Devastatingly human: an analysis of registered nurses' medication error accounts. Qual Health Res. 2010;20(10):1327-42.

43. Treiber LA, Jones JH. Medication errors, routines, and differences between perioperative and non-perioperative nurses. AORN J. 2012;96(3):285-94.

44. Taxis K, Barber N. Incidence and severity of intravenous drug errors in a German hospital. Eur $\mathbf{J}$ Clin Pharmacol. 2004;59(11):815-7.

45. Jones JH, Treiber L. When the 5 rights go wrong: medication errors from the nursing perspective. J Nurs Care Qual. 2010;25(3):240-7.

46. Dean B, Barber N. The effects of a patients' own drugs scheme on the incidence and severity of medication administration errors. Int J Pharm Prac. 2000;8(3):209-16.

47. Ho CYW, Dean BS, Barber ND. When do medication administration errors happen to hospital inpatients? Int J Pharm Prac. 1997;5(2):91-6.

48. Cavell GF, Hughes DK. Does computerized prescribing improve the accuracy of drug administration? Pharm J. 1997;259(6968):782-4.

49. Bruce J, Wong I. Parenteral drug administration errors by nursing staff on an acute medical admissions ward during day duty. Drug Saf. 2001;24(11):855-62.

50. Hartley GM, Dhillon S. Observational study of the prescribing and administration of intravenous drugs in a general hospital. Int J Pharm Prac. 1998;6(1):38-45.

51. Hand K, Barber N. Nurses' attitudes and beliefs about medication errors in a UK hospital. Int J Pharm Prac. 2000;8:128-34.
52. Gladstone J. Drug administration errors: a study into the factors underlying the occurrence and reporting of drug errors in a district general hospital. J Adv Nurs. 1995;22(4):628-37.

53. Sanghera IS, Franklin BD, Dhillon S. The attitudes and beliefs of healthcare professionals on the causes and reporting of medication errors in a UK intensive care unit. Anaesthesia. 2007;62(1):53-61.

54. Balas MC, Scott LD, Rogers AE. The prevalence and nature of errors and near errors reported by hospital staff nurses. Appl Nurs Res. 2004;17(4):224-30.

55. Taylor JA, Loan LA, Kamara J, et al. Medication administration variances before and after implementation of computerized physician order entry in a neonatal intensive care unit. Pediatrics. 2008;121(1):123-8.

56. Leape LL, Bates DW, Cullen DJ, et al. Systems analysis of adverse drug events. ADE Prevention Study Group. JAMA. 1995;274(1):35-43.

57. Cullen D, Sweitzer BJ, Bates DW, et al. Preventable adverse drug events in hospitalized patients: a comparitive study of intensive care and general care units. Crit Care Med. 1997;25(8):1289-97.

58. Balas MC, Scott LD, Rogers AE. Frequency and type of errors and near errors reported by critical care nurses. Can J Nurs Res. 2006;38(2):24-41.

59. Colligan L, Bass EJ. Interruption handling strategies during paediatric medication administration. BMJ Qual Saf. 2012;21(11):912-7.

60. Stetina P, Groves M, Pafford L. Managing medication errors-a qualitative study. Medsurg Nurs. 2005;14(3):174-8.

61. Haghenbeck KT. Critical care nurses' experiences when technology malfunctions. J N Y State Nurses Assoc. 2005(SpringSummer);36(1):13-9.

62. Nichols P, Copeland TS, Craib IA, et al. Learning from error: identifying contributory causes of medication errors in an Australian hospital. Med J Aust. 2008;188(5):276-9.

63. Reid-Searl K, Moxham L, Happell B. Enhancing patient safety: the importance of direct supervision for avoiding medication errors and near misses by undergraduate nursing students. Int $\mathrm{J}$ Nurs Pract. 2010;16(3):225-32.

64. Deans C. Medication errors and professional practice of registered nurses. Collegian. 2005;12(1):29-33.

65. Gill F, Corkish V, Robertson J, et al. An exploration of pediatric nurses' compliance with a medication checking and administration protocol. J Spec Pediatr Nurs. 2012;17(2):136-46.

66. Manias E, Aitken R, Dunning T. Medication management by graduate nurses: before, during and following medication administration. Nurs Health Sci. 2004;6(2):83-91.

67. Baker HM. Rules outside the rules for administration of medication: a study in New South Wales, Australia. Image J Nurs Sch. 1997;29(2):155-8.

68. Gordon PC, Llewellyn RL, James MF. Drug administration errors by South African anaesthetists-a survey. S Afr Med J. 2006;96(7):630-2.

69. Llewellyn RL, Gordon PC, Wheatcroft D, et al. Drug administration errors: a prospective survey from three South African teaching hospitals. Anaesth Intensive Care. 2009;37(1):93-8.

70. Labuschagne M, Robbetze W, Rozmiarek J, et al. Errors in drug administration by anaesthetists in public hospitals in the Free State. S Afr Med J. 2011;101(5):324-7.

71. Gordon PC. Wrong drug administration errors amongst anaesthetists in a South African teaching hospital. South Afr J Anaesth Analg. 2004;10(2):7-8.

72. Webster CS, Merry AF, Larsson L, et al. The frequency and nature of drug administration error during anaesthesia. Anaesth Intensive Care. 2001;29(5):494-500. 
73. McBride-Henry K, Foureur M. A secondary care nursing perspective on medication administration safety. J Adv Nurs. 2007;60(1):58-66.

74. Dickinson A, McCall E, Twomey B, et al. Paediatric nurses' understanding of the process and procedure of double-checking medications. J Clin Nurs. 2010;19(5-6):728-35.

75. Orser BA, Chen RJ, Yee DA. Medication errors in anesthetic practice: a survey of 687 practitioners. Can J Anaesth. 2001;48(2):139-46.

76. Hall KW, Ebbeling P, Brown B, et al. A retrospective-prospective study of medication errors: basis for an ongoing monitoring program. Can J Hosp Pharm. 1985;38(5):141-3,146.

77. Chua SS, Tea MH, Rahman MH. An observational study of drug administration errors in a Malaysian hospital (study of drug administration errors). J Clin Pharm Ther. 2009;34(2):215-23.

78. Chua SS, Chua HM, Omar A. Drug administration errors in paediatrics wards: a direct observation approach. Eur J Paediatr. 2010;169(5):603-11.

79. van Gijssel-Wiersma DG, van den Bemt PM, Walenbergh-van Veen MC. Influence of computerised medication charts on medication errors in a hospital. Drug Saf. 2005;28(12):1119-29.

80. Rodriguez-Gonzalez CG, Herranz-Alonso A, Martin-Barbero ML, et al. Prevalence of medication administration errors in two medical units with automated prescription and dispensing. J Am Med Inform Assoc. 2012;19(1):72-8.

81. Rai E. Medication errors in a tertiary care institution-an audit. J Anaesth Clin Pharmacol. 2010;26(3):371-4.

82. Tang FI, Sheu SJ, Yu S, et al. Nurses relate the contributing factors involved in medication errors. $J$ Clin Nurs. 2007;16(3):447-57.

83. Vazin A, Delfani S. Medication errors in an internal intensive care unit of a large teaching hospital: a direct observation study. Acta Med Iran. 2012;50(6):425-32.

84. Agalu A, Ayele Y, Bedada W, et al. Medication administration errors in an intensive care unit in Ethiopia. Int Arch Med. 2012;5(1):15.

85. Kim KS, Kwon SH, Kim JA. Nurses' perceptions of medication errors and their contributing factors in South Korea. J Nurs Manag. 2011;19(3):346-53.

86. Zhang Y, Dong YJ, Webster CS, et al. The frequency and nature of drug administration error during anaesthesia in a Chinese hospital. Acta Anaesthesiol Scand. 2013;57(2):158-64.

87. Fasting S, Gisvold SE. Adverse drug errors in anaesthesia, and the impact of coloured syringe labels. Can J Anaesth. 2000;47(11):1060-7.

88. Ozkan S, Kocaman G, Ozturk C, et al. Frequency of pediatric medication administration errors and contributing factors. J Nurs Care Qual. 2011;26(2):136-43.

89. No authors listed. ASHP Standard definition of a medication error. Am J Hosp Pharm. 1982;39(2):321.

90. No authors listd. ASHP guidelines on preventing medication errors in hospitals. Am J Hosp Pharm. 1993;50(2):305-14.

91. Bryman A. Social research methods. 4th rev ed. New York: Oxford University Press; 2012.

92. Dornan T, Ashcroft D, Heathfield H, et al. An in depth investigation into causes of prescribing errors by foundation trainees in relation to their medical education. EQUIP study. General Medical Council 2009. Available from URL http://www.gmcuk.org/FINAL_Report_prevalence_and_causes_of_prescribing errors.pdf_28935150.pdf. Accessed 2013 July 2.
93. Paulhus D. Measurement and control of response bias. In: Robinson J, Shaver P, Wrightsman L, editors. Measures of personality and social psychological attitudes. San Diego: Academic Press; 1991. p. 17-59.

94. Nguyen C, Côté J, Lebel D, et al. The AMÉLIE project: failure mode, effects and criticality analysis: a model to evaluate the nurse medication administration process on the floor. J Eval Clin Pract. 2013;19(1):192-9.

95. Shebl N, Franklin B, Barber N, et al. Failure mode and effects analysis: views of hospital staff in the UK. J Health Serv Res Policy. 2012;17(1):37-43.

96. Young J, Slebodnik M, Sands L. Bar code technology and medication administration error. J Patient Saf. 2010;6(2):115-20.

97. Wulff K, Cummings GG, Marck P, et al. Medication administration technologies and patient safety: a mixed-method systematic review. J Adv Nurs. 2011;67(10):2080-95.

98. Hsaio GY, Chen IJ, Yu S, et al. Nurses' knowledge of high-alert medications: instrument development and validation. J Adv Nurs. 2010;66(1):177-90.

99. Alsulami Z, Conroy S, Choonara I. Double checking the administration of medicines: what is the evidence? A systematic review. Arch Dis Child. 2012;97(9):833-7.

100. Phipps E, Turkel M, Mackenzie ER, et al. He thought the "lady in the door" was the "lady in the window": a qualitative study of patient identification practices. Jt Comm J Qual Patient Saf. 2012;38(3):127-34.

101. Alper SJ, Holden RJ, Scanlon MC, et al. Self-reported violations during medication administration in two paediatric hospitals. BMJ Qual Saf. 2012;21(5):408-15.

102. Reckmann MH, Westbrook JI, Koh Y, et al. Does computerised provider order entry reduce prescribing errors for hospital inpatients? A systematic review. J Am Med Inform Assoc. 2009; 16:613-23.

103. Franklin BD, O'Grady K, Donyai P, et al. The impact of a closed-loop electronic prescribing and administration system on prescribing errors, administration errors and staff time: a beforeand-after study. Qual Saf Health Care. 2007;16:279-84.

104. Hughes RG (ed). Patient safety and quality: an evidence-based handbook for nurses. Rockville, MD: US Agency for Healthcare Research and Quality; 2008.

105. Potter P, Wolf L, Boxerman S, et al. Understanding the cognitive work of nursing in the acute care environment. J Nurs Adm. 2005; 35(7-8):327-35.

106. Biron A. Medication administration complexity, work interruptions, and nurses' workload as predictors of medication administration errors [thesis]. Canada: McGill University; 2009.

107. Scott-Cawiezell J, Pepper GA, Madsen RW, et al. Nursing home error and level of staff credentials. Clin Nurs Res. 2007;16(1):72-8.

108. Westbrook JI, Woods A, Rob MI, et al. Association of interruptions with an increased risk and severity of medication administration errors. Arch Intern Med. 2010;170(8):683-9097.

109. Buchini S, Quattrin R. Avoidable interruptions during drug administration in an intensive rehabilitation ward: improvement project. J Nurs Manag. 2012;20(3):326-34.

110. Aspden P, Wolcott JA, Bootman JL, et al., editors. Preventing medication errors. Washington DC: National Academies Press; 2007.

111. Vincent C. Patient safety. 2nd rev ed. Chichester: WileyBlackwell; 2010: 273. 